\title{
Review of coal-water fuel pulverization technology and atomization quality registration methods
}

\author{
Andrey Zenkov ${ }^{1, *}$, Kirill Larionov ${ }^{1}$, Vladimir Gubin ${ }^{1}$ \\ ${ }^{1}$ National Research Tomsk Polytechnic University, 634050 Tomsk, Russia
}

\begin{abstract}
Possibilities of coal-water fuel application in industrial power engineering are considered and described. Two main problems and disadvantages of this fuel type are suggested. The paper presents information about liquid fuel atomization technologies and provides data on nozzle type for coal-water fuel pulverization. This article also mentions some of the existing technologies for coal-water slurry spraying quality determination.
\end{abstract}

\section{Coal-water fuel}

Technologically advanced countries are conducting efforts to improve coal fuel utilization technologies to provide an increase of their ecological and economical parameters, as well as reliable and safe power equipment operation [1]. One of the advanced solutions in energy industry is development and implementation of coal-water fuel technology (CWF) [2].

CWF represents composite liquid fuel: mixture of pulverized coal and water with or without surfactants. It is capable of replacing gas and oil at boiler stations and TPPs [3].

CWF technology is not only a way of coal transportation through pipelines, but also a way of coal sludge recycling. These sludges are now recycled as waste, but they can be used for suspension preparation and used as fuel at power generating facilities. Moreover, moistened sludges are actually a semi-finished product for CWF preparation, which reduces cost of slurry production. It would be more economically advantageous to use coal-water fuel prepared from sludges directly on coal-mining fields to provide their auxiliary power. However, due to convenience of CWF transporting (in tanks or pipelines), it is possible to use this technology at energy facilities.

It is also important to note ecological aspect of sludge accumulation. There is already huge amount of waste in places where coal is extracted and enriched, and this figure continues to grow every day. For example, the Kemerovo Region (95.5 thousand square kilometers) occupies the first place in Russia in terms of industrial waste generation. More than 700 thousand tons of solid wastes are produced only in Kemerovo annually, most of which are sludges from coal industry. Area of disturbed land near the city is 500 hectares. Thus, processing of coal slurries will allow using them as raw materials, reducing the

\footnotetext{
*Corresponding author: andreyzenkov@mail.ru
} 
amount of waste to a minimum. Clearance of the areas occupied today by this type of waste will allow reducing man-caused impact on the environment and amount of dust in the air [4].

The main advantages of coal-water fuel (comparing to coal) are:

- high burnout degree - up to $99 \%$;

- possibility to use both high-quality and low-grade coal;

- large emission improvement of nitrogen oxides, carbon monoxide and fly ash;

- utilization of coal-preparation plants wastes (coal slack);

- fire and explosion safety;

- convenience of transportation (by pipe line, in tankers) and storage (no dusting).

Besides CWF application for direct combustion in boilers, there is a possibility of its gasification and further implementation of the obtained synthesis gas for energy production [5]. For example, it can be used at Combined Cycle Gas Turbine Plant with triple cycle in extreme north regions [6].

However, considering advantages listed above, CWF technology has some unsolved problems which influence its application in energy industry. First of all it concerns nozzles operation [7], which is affected by initial fuel metamorphic properties, granulometric and concentration compositions because an increase of coal part in CWF leads to significant increase of viscosity. It, in turn, causes combustion efficiency reduction [8]. It is proved [9] that viscosity hinges on coal concentration in CWS. Consequently, it is necessary to decrease coal concentration to obtain suspension with an acceptable viscosity, which leads to CWF calorific value decrease. Therefore, the main obstacle of coal-water fuel common usage is its rheological properties (viscosity and sedimentation stability).

\section{Existing technologies of coal-water fuel atomization}

There are many different technologies for pulverization of liquid fuel including coal-water slurry. They are hydraulic, mechanical, pneumatic, acoustic atomization and pulverization with radial-spray nozzles. Each of these methods is divided into several subtypes [10].

Atomization efficiency is strongly influenced by correct choice of pulverization device. It is believed, that it is viable to implement mechanical and pneumatic nozzles for coalwater fuel atomization because this type of fuel is highly viscous. Now, combination of these two nozzle types is applied for CWF injection into a combustion chamber pneumatic-mechanical (fig. 1).

These injectors operate with high fuel pressure in nominal mode, but at low flow rate with supply of spray agent (air). The initial spraying is conducted in mechanical nozzle, and then additionally with compressed air, which is fed through the holes of swirler. In most designs, tangential speed is imparted to the fuel. Atomizer is constructed by one of the simplest schemes of centrifugal nozzles: with circular or rectangular input channels, with distributing washer or without it, etc. Such injectors are divided into two types: with external and internal interaction of flows [11].

However, due to CWF unstable rheological characteristics and presence of coal particles in its composition these atomizers are exposed to erosive wear, which in turn leads to necessity of this technology improvement.

\section{Methods of atomization quality analysis}

Stability and completeness of fuel combustion in combustion chamber are characterized by degree of its atomization and perfection of gas flow aerodynamics inside the chamber. These qualities provide reliable conditions for ignition and combustion of spray cone, 
reliable mixing of fuel and oxidizer and temperature distribution, as well as optimal conditions for burning fuel throughout the chamber. Quality of coal-water suspension atomization in combustion chamber depends on fuel properties and design of atomizing device [12].

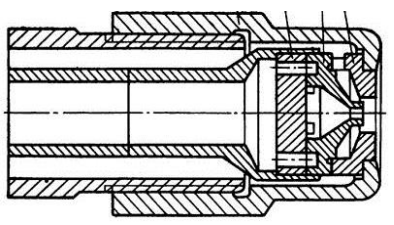

Fig. 1. Pneumatic-mechanical atomizer.

It is pointed out in work [13] that coal-water fuel combustion differs significantly from pulverized coal burning process.

In [7], it was suggested that when coal-water suspension is sprayed, a polydisperse droplet stream is formed which contains only carbon particles "freed" from liquid phase due to high speed and drops of coal-water fuel consisting of fine coal particles and liquid phase. At the same time, the amount of coal particles in CWS can reach 25-30\%, depending on particle size distribution.

According to [12], spraying of coal-water fuel with air or steam is conducted in two stages. In the first stage, when CWS and atomizing agent mixing occurs in the nozzle, jet of suspension is crushed due to kinetic energy of the latter. At the second stage, crushing of CWS drops happens due to resistance forces of surrounding gaseous medium, velocity of which is many times less than speed of droplet. As a result of dynamic effects, droplets are flattened and torn into smaller ones.

Paper [7] represents experimental results on CWF pulverization with pneumaticmechanical atomizer. Special test-bench was created for it (fig. 2). CWF pulverization was conducted onto a screen with tray. Then configuration of spray cone was determined: it was intercepted by aluminum platen; atomization quality was defined by the print on the platen.

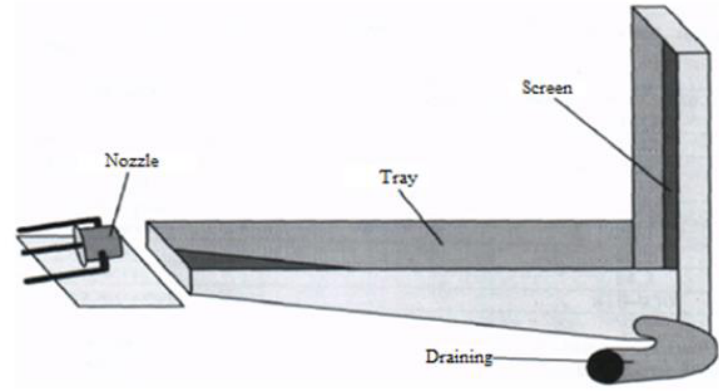

Fig 2. Test bench for coal-water fuel pulverization.

However, this method of atomization quality registration is obsolete and inexact. Nowadays, there are new methods for shaded flows atomization analysis.

One of them is PIV-method (Particle Image Velocimetry). Small particles, called tracers, which are placed in the flow, are used in this method. Their speed and direction are defined by a laser and a high speed camera [14].

Daviault et al. [15] used laser-Doppler anemometry (LDA) to determine quantitative characteristics of pulverization. LDA is noncontacting measurement method of direction and velocity of suspended particulate matters in a liquid or gas flow. It is based on two convergent laser beams directed at the flow so that they intersect in the flow and form control measurement area. Interference fringes are formed in this area (fig. 3). The distance between them can be calculated. Particles intersect these fringes and produce light pulses, 
the time of which is registered. Then, knowing distance and time, velocity of particles in the flow is calculated $[16,17]$.

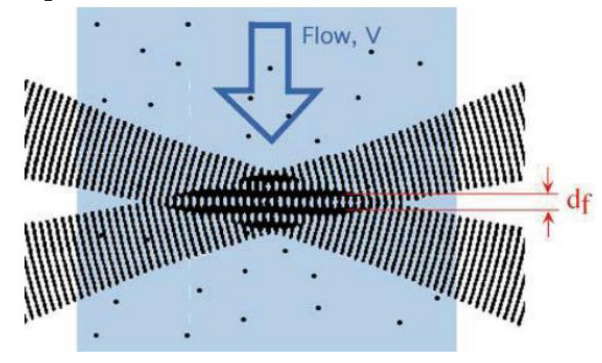

Fig 3. Control measurement area with interference fringes.

\section{Conclusion}

Conducted review showed that application of coal-water fuel in power engineering is a promising direction. However, there are several unsolved issues associated with CWF production, storage and pulverization. Therefore, one of the most urgent goals in this area is development of more effective atomizers, as well as evaluation and selection of CWF production method in each specific case due to nonuniformity of coal characteristics even within one grade.

The research has been accomplished with financial support of the Russian Federation Ministry of Education and Science within implementation of the project N 13.7644.2017/BCH.

\section{References:}

1. B.M. Vorobiev, Bulletin of Russian academy of sciences, 11, 65 (2011) [in Russian]

2. G.S. Hodakov, Therm. Eng. 54, 36 (2007)

3. Y. Wei, J. Wang, Fuel Proc. Tech. 143, 18 (March 2016)

4. E.S. Zlobina, A.V. Papin, A.Yu. Ignatova, IV International Scientific and Practical Conference, 213 (2015) [in Russian]

5. K.V. Slyusarskiy, K.B. Larionov, V.I. Osipov, V.E. Gubin, A.A. Gromov, Fuel 191, $383(2017)$

6. N. Galashov, S. Tsibulskii, A. Matveev, V. Masjuk, EPJ Web Conf. 110, 01019 (2016)

7. U.A. Senchurova, V.I. Murko, V.I. Fedyayev, D.A. Dzyuba, E.M. Puzyrev, Bulletin Tomsk polytech. univ. 312, 37 (2008) [in Russian]

8. M.P. Baranova, V.A. Kulagin (Siberian Federal University, 2011) [in Russian]

9. K.B. Larionov, A.V. Zenkov, S.A. Yankovsky, A.A. Ditc, IFOST 2016. 7884323, 568 (Novosibirsk, 2017)

10. D.G. Paji, V.S. Galustov, Atomizers of liquid (Chemistry, Moscow, 1979) [in Russian]

11. L.V. Kulagin, M.Ya. Moroshkin, Nozzles for spraying heavy fuels (Mashinostroenie, Moscow 1973) [in Russian]

12. U.A. Senchurova, E.V. Zarechneva, Inf. tech. 122 (2012) [in Russian]

13. B.N. Smetannikov, Investigation of ignition and combustion of coal-water suspension drop, 556 (1969) [in Russian]

14. R. Ben Haj Slama, B. Gilles, M. Ben Chiekh, J-C. Bera, Ultrasonics, 76, 217 (2017)

15. S.G. Daviault, O.B. Ramadan, E.A. Matida, P.M. Hughes, R. Hughes, Fuel, 98, 183 (2012)

16. J.J. Chinn, D Cooper., A.J. Yule, G.G. Nasr, Heat Mass Trans. 52, 2037 (2016)

17. S.V. Poplavski, V.M. Boiko, A.U. Nesterov, ICMAR 2016. 1770, 030016 (2016) 